TRANSACTIONS OF THE

AMERICAN MATHEMATICAL SOCIETY

Volume 349, Number 8, August 1997, Pages 3093-3104

S 0002-9947(97)01936-3

\title{
THE SECOND VARIATION OF NONORIENTABLE MINIMAL SUBMANIFOLDS
}

\author{
MARTY ROSS
}

\begin{abstract}
Suppose $M$ is a complete nonorientable minimal submanifold of a Riemannian manifold $N$. We derive a second variation formula for the area of $M$ with respect to certain perturbations, giving a sufficient condition for the instability of $M$. Some simple applications are given: we show that the totally geodesic $\mathbb{R P}^{2}$ is the only stable surface in $\mathbb{R} \mathbb{P}^{3}$, and we show the non-existence of stable nonorientable cones in $\mathbb{R}^{4}$. We reproduce and marginally extend some known results in the truly non-compact setting.
\end{abstract}

\section{INTRODUCTION}

Suppose $M$ and $N$ are complete and connected Riemannian manifolds and suppose $\Psi: M \rightarrow N$ is an isometric minimal immersion. Let $V$ be a smooth, compactly supported vector field along $M$, the "initial velocity" of a perturbation of $M$. (More precisely, $V$ is a section of the pull-back bundle on $M$ obtained from $N$ via $\Psi$, giving a perturbation of $\Psi$; to avoid such cumbersome expressions, we shall think of $M$ lying in $N$ and suppress mention of the immersion $\Psi$.) Since $M$ is minimal, the first variation of the area of $M$ with respect to $V$ is zero. The second variation is given by ([LaS, Th1], [Si1])

$$
\begin{aligned}
A^{\prime \prime}(V)= & \int_{M} \sum_{i=1}^{n}\left|\nabla_{\tau_{i}}^{\perp} V\right|^{2}+\left(\operatorname{div}_{M} V\right)^{2}-\sum_{i, j=1}^{n}\left\langle\tau_{i}, \nabla_{\tau_{j}} V\right\rangle\left\langle\tau_{j}, \nabla_{\tau_{i}} V\right\rangle \\
& -\sum_{i=1}^{n} R\left(\tau_{i}, V, V, \tau_{i}\right) .
\end{aligned}
$$

Here, $\langle$,$\rangle is the metric on N, \operatorname{dim} M=n, \nabla$ is the Riemannian connection on $N, \perp$ indicates the orthogonal complement to the tangent bundle $T M$ in $T N, \tau_{1}, \ldots, \tau_{n}$ is an orthonormal basis for $T_{p} M$ (for any given $p \in M$ ), $\operatorname{div}_{M} V \equiv \sum_{i}\left\langle\tau_{i}, \nabla_{\tau_{i}} V\right\rangle$, and $R$ is the Riemannian curvature tensor on $N$. We say $M$ is stable if $A^{\prime \prime}(V) \geq 0$ for every such $V$.

In certain contexts one can hope to give a complete classification of the stable submanifolds of $N$, and there has been much recent research devoted to obtaining such "Bernstein theorems" - see [Ca1] for a short survey and some references. Commonly, there is an implicit or explicit assumption that the submanifold $M$ be orientable; most often $N$ is also assumed to be orientable, and $M$ is assumed to be

Received by the editors July 21, 1994.

1991 Mathematics Subject Classification. Primary 53C45; Secondary 58E12.

Key words and phrases. Nonorientable minimal surface, stable, Bernstein Theorem, second variation. 
of codimension 1 in $N$ (note as exceptions [Sy, Th1], [Sm, Th5.1.1], [LaS, Cor1, 8], $[\mathrm{Mi}])$. With these extra hypotheses there exists a smooth unit normal $\nu$ globally defined on $M$, and it is then sufficient ([Si1, p19]) to consider variations of the form

$$
V=\phi \nu
$$

where $\phi: M \rightarrow \mathbb{R}$ is a smooth, compactly supported test-function. (1) then simplifies to

$$
A^{\prime \prime}(\phi)=\int_{M}\left|\nabla^{M} \phi\right|^{2}-\left(|B|^{2}+\operatorname{Ric}(\nu)\right) \phi^{2},
$$

where $\nabla^{M}$ is the connection on $M,|B|^{2} \equiv \sum_{i, j}\left|\nabla_{\tau_{i}}^{\perp} \tau_{j}\right|^{2}$ is the norm-squared of the second fundamental form of $M$, and $\operatorname{Ric}(\nu)=\sum_{i} R\left(\tau_{i}, \nu, \nu, \tau_{i}\right)$ is the Ricci curvature of $N$ in the direction $\nu$. This form of the second variation is of course much easier to interpret and to apply. We note especially the application of (2) in [FS], where it is proved that there are very strong restrictions on stable, orientable $M$ when $N$ is a 3-manifold of nonnegative scalar curvature ([FS, Th3]) - in particular, it is proved there that if $N=\mathbb{R}^{3}$ then the only stable orientable submanifolds are flat planes ([FS, Cor4]).

The number of articles which allow or assume $M$ to be nonorientable is small. Apart from our own work, cited below, we are aware of the following results:

(a) $[\mathrm{Sm}, \mathrm{Th} 5.1 .1],[\mathrm{LaS}, \mathrm{Cor} 1]$ prove the non-existence of stable submanifolds (more generally, integral varifolds) of the unit sphere $\mathbb{S}^{n}$. They take as $V$ the projection into $T \mathbb{S}^{n}$ of constant vector fields in $\mathbb{R}^{n+1}$ and apply (1).

(b) [Ch, Th6], [LiS, Cor8] prove that if $M \subset \mathbb{R}^{3}$ is a finitely punctured projective plane or Klein bottle with finite total curvature then $M$ is unstable. As in (a), the proofs use constant vector fields in the ambient space, here to show the existence of a Jacobi field on a compact portion of $M$.

(c) $[\mathrm{LiS}]$ also establishes the instability of nonorientable $M \subset \mathbb{R}^{3}$ with infinite total curvature and finite topology. Outside of a compact set $M$ is orientable, permitting the use of (2), and thus the results of [F] can be applied. (The same argument applies if $\mathbb{R}^{3}$ is replaced by any 3-manifold $N$ with nonnegative scalar curvature).

(d) Similar to (c), [Yp, Th3.1] makes the assumption that $M$ is "two-sided" in $N$, permitting application of (2). Arguing as in $[\mathrm{P}]$, this permits a generalization of [FS, Th3].

It is not difficult to find reasons for this scarcity of results in the nonorientable setting. To begin, it is only in special cases that the ambient manifold provides us with natural (Killing) vector fields $V$ to use in (1), and even then the space of such fields is small. On the other hand, (2) is not available to us except in very special circumstances. Moreover, the existence in certain contexts of non-trivial stable nonorientable surfaces (see below, and §3) suggests that these are not just technical difficulties: it really is easier for a nonorientable minimal submanifold to be stable. Nonetheless, there are definitely contexts in which one expects there to be few if any stable nonorientable submanifolds (when $N=\mathbb{R}^{3}$, for instance), and a more systematic approach is certainly desirable.

In [R1, Th3] we generalized (b), proving that if $M$ is a complete nonorientable minimal surface of finite total curvature in $\mathbb{R}^{3}$ then $M$ is unstable. The approach there was to consider symmetric variations of the orientable double cover $\widetilde{M}$ of $M$, 
allowing the use of (2) for antisymmetric functions on $\widetilde{M}$. The idea then was to use in (2) the components of a low energy map $F: \widetilde{M} \rightarrow \mathbb{S}^{2} \subset \mathbb{R}^{3}$, a technique used previously in similar contexts (see, for example, [LY, Th1], [Ya, p126], [LR, Th2], [RR, Th4], [EI, Cor2.8]). This approach was used again in [RS, Cor2] to study the quotients of periodic minimal surfaces in $\mathbb{R}^{3}$ of finite total curvature: it was proved there that the only such stable singly-periodic surface is the helicoid and the only stable doubly-periodic surfaces are Sherk's surfaces.

Our intention here is to generalize the argument outlined in the previous paragraph. In $\S 2$ we consider a special case of (1) for nonorientable $M$, introducing a "penalization" associated with a map $F: \widetilde{M} \rightarrow \mathbb{S}^{2}$ (Proposition 1). The resulting expression $\left(1^{\prime}\right)$ gives a sufficient condition for the instability of $M$. In the special case when $M$ is a hypersurface of an orientable manifold $N$, we obtain a generalization $\left(2^{\prime}\right)$ of (2) which applies to any test-function $\phi: M \rightarrow \mathbb{R}$.

The underlying philosophy is this: if the related orientable problem is well understood and if we can find a satisfactory map $F$, then we may be able to say something about the nonorientable problem. Of course, neither of these "ifs" is trivial; in particular, the only case in which we know of half-way decent candidates for $F$ is when $M$ is compact and 2-dimensional (one should include $M$ having finite total curvature under this heading, as discussed in $\S 2$ ): the maps here are given by [Ma, p481], and will be our source for the rest of the paper (Lemma 2). Concentrating on the compact setting, we show in $\S 3$ that the totally geodesic $\mathbb{R} \mathbb{P}^{2}$ is the only stable surface in $\mathbb{R P}^{3}$ (Proposition 3 ), and we show in $\S 4$ that there are no stable nonorientable cones in $\mathbb{R}^{4}$ (Proposition 6).

It is readily apparent that there are limitations to the proposed method, and we suggest it less as a panacea for the nonorientable than merely as the best current choice from an unpromising lot. There are severe problems, even in the compact 2-dimensional case, and it is not at all clear how to approach the truly noncompact and higher dimensional cases. In $\S 5$ we take some tentative steps towards addressing the noncompact case, considering nonorientable immersions into 3-manifolds of nonnegative scalar curvature. We use the arguments of [GL, §3] to recover and slightly generalize (c) (Propositions 7,8).

\section{A Second Variation Formula}

Suppose $M$ is a nonorientable submanifold of $N$ and let $\widetilde{M}$ be the orientable double cover of $M$ (see, e.g. [H, Th2.12], [Ca2, p34]). Associated to $\widetilde{M}$ is the $2: 1$ projection $\pi: \widetilde{M} \rightarrow M$ and the antipodal map $I: \widetilde{M} \rightarrow \widetilde{M}$, an orientation-reversing fixed-point-free isometric involution satisfying $\pi \circ I=\pi$. A vector field $V$ on $\widetilde{M}$ projects to a vector field on $M$ iff

$$
V \circ I=V
$$

and, conversely, every vector field on $M$ lifts to a vector field on $\widetilde{M}$ satisfying (3). It is thus easy to show that studying variations of $M$ is equivalent to studying variations of $\widetilde{M}$ by fields satisfying (3).

Suppose, now, that $W$ is an antisymmetric vector field on $\widetilde{M}$,

$$
W \circ I=-W,
$$


and that $f: M \rightarrow \mathbb{R}$ is also antisymmetric,

$$
f \circ I=-f \text {. }
$$

Then the product $V=f W$ satisfies (3) and so may be substituted in (2). We are interested in applying this when given a map

$$
F=\left(f_{1}, \ldots, f_{l}\right): \widetilde{M} \rightarrow \mathbb{S}^{l-1} \subset \mathbb{R}^{l}, \quad l \geq 2,
$$

for which each component is antisymmetric. Then, taking variations $f_{\alpha} W$ for $\alpha=$ $1, \ldots, l$ and summing, a standard calculation gives:

Proposition 1 (Second Variation Formula).

$$
\begin{aligned}
\left(1^{\prime}\right) \sum_{\alpha=1}^{l} A^{\prime \prime}\left(f_{\alpha} W\right)= & A^{\prime \prime}(W)+\int_{\widetilde{M}}|\widetilde{\nabla} F|^{2}\left|W^{\perp}\right|^{2} \\
= & \int_{\widetilde{M}} \sum_{i=1}^{n}\left|\nabla_{\tau_{i}}^{\perp} W\right|^{2}+\left(\operatorname{div}_{\widetilde{M}} W\right)^{2}-\sum_{i, j=1}^{n}\left\langle\tau_{i}, \nabla_{\tau_{j}} W\right\rangle\left\langle\tau_{j}, \nabla_{\tau_{i}} W\right\rangle \\
& +\int_{\widetilde{M}}-\sum_{i=1}^{n} R\left(\tau_{i}, W, W, \tau_{i}\right)+|\widetilde{\nabla} F|^{2}\left|W^{\perp}\right|^{2} .
\end{aligned}
$$

Here $|\widetilde{\nabla} F|^{2} \equiv \sum_{\alpha} \sum_{i}\left(\tau_{i}\left(f_{\alpha}\right)\right)^{2}$ is the energy density of $F$.

The obvious consequence of $\left(1^{\prime}\right)$ is to give a sufficient condition for the instability of $M$ : for any choice of antisymmetric $W$ and $F$

$$
A^{\prime \prime}(W)+\int_{\widetilde{M}}|\widetilde{\nabla} F|^{2}\left|W^{\perp}\right|^{2}<0 \quad \Longrightarrow \quad M \text { is unstable. }
$$

Of course for this implication to be of any use, we need candidates for $W$ and $F$. We begin to specialize by assuming that the ambient manifold $N$ is orientable and that $M$ is codimension 1 in $N$. Then we can choose a smooth unit normal $\nu$ globally on $\widetilde{M}$, and we note that $\nu$ will satisfy (4). Next, suppose $\phi: M \rightarrow \mathbb{R}$ is any smooth, compactly supported function on $M$ and let $\widetilde{\phi}=\phi \circ \pi: \widetilde{M} \rightarrow \mathbb{R}$ be the lift of $\phi$. Then $W=\widetilde{\phi} \nu$ also satisfies (4). Thus, for any choice of antisymmetric $F$, we have legitimate variations $f_{\alpha} \tilde{\phi} \nu$, and

$$
\begin{aligned}
\sum_{\alpha=1}^{l} A^{\prime \prime}\left(f_{\alpha} \widetilde{\phi}\right) & =\int_{\widetilde{M}}|\widetilde{\nabla} \widetilde{\phi}|^{2}-\left(|B|^{2}+\operatorname{Ric}(\nu)\right)(\widetilde{\phi})^{2}+|\widetilde{\nabla} F|^{2}(\widetilde{\phi})^{2} \\
& =2 \int_{M}\left|\nabla^{M} \phi\right|^{2}-\left(|B|^{2}+\operatorname{Ric}(\nu)\right) \phi^{2}+|\widetilde{\nabla} F|^{2} \phi^{2}
\end{aligned}
$$

(Note that $|\widetilde{\nabla} F|^{2}$ is well-defined on $M$.)

We now confront the second and more serious of our problems, that of finding suitable maps $F$; of course, to be of any use, $|\widetilde{\nabla} F|^{2}$ must be at least comparable to $|B|^{2}+\operatorname{Ric}(\nu)$. As mentioned in $\S 1$, the only case we know of where there are candidates for $F$ is when $M$ is (essentially) compact and 2-dimensional. Restricting to oriented isothermal coordinates, $\widetilde{M}$ is then a compact Riemann surface. We can thus apply 
Lemma 2 ([Ma, p481]). Suppose $\widetilde{M}$ is a compact Riemann surface of genus $\gamma$ with antipodal map $I: \widetilde{M} \rightarrow \widetilde{M}$. Then there is an antisymmetric and conformal $F: \widetilde{M} \rightarrow$ $\mathbb{S}^{2}$ with

$$
\operatorname{deg} F \leq \gamma+1
$$

Consequently, for any metric compatible with the conformal structure of $\widetilde{M}$,

$$
\int_{\widetilde{M}}|\widetilde{\nabla} F|^{2}=8 \pi(\operatorname{deg} F) \leq 8 \pi(\gamma+1) .
$$

Lemma 2 will be our source of maps in the sections to follow; here, we end with some comments.

(a) [LY, p272] claims a result along the lines of Lemma 2, but the argument there appears to be invalid.

(b) If $M$ has finite total curvature then $\widetilde{M}$ is conformally a finitely punctured compact Riemann surface. So, choosing $\phi$ to be a suitable cut-off function as in [F, p131], we can apply $F$ as given in the lemma. This is essentially the argument used in [R1, Th3] and [RS, Cor2].

(c) In general, the estimate (6) is the best we can hope for ([Ma], [R1, p73]). It is not clear whether or when the estimate (7) can be lowered by the consideration of non-conformal maps.

(d) If one does not demand antisymmetry then $F$ can be chosen to have degree $\leq \frac{1}{2}(\gamma+3)$ (see [GH, pp261,358]). This better estimate is used in [Ya, p126], [RR, Th4], [EI, Cor2.8], and is the reason that the results there are more definitive than our own below.

\section{Compact Surfaces in 3-Manifolds of Nonnegative Ricci Curvature}

[FS, Th3] essentially classifies the stable orientable surfaces in 3-manifolds of nonnegative scalar curvature, and genus bounds can be obtained for orientable index one minimal surfaces and stable constant mean curvature surfaces in 3-manifolds of nonnegative Ricci curvature ([Ya, p126], [EI, Cor2.8], [RR, p294]). We can give no results of comparable strength for nonorientable surfaces (see Remark (c) below), but a little information can be obtained. Here we investigate the situation for compact surfaces; we study noncompact surfaces in $\S 5$.

If $M$ is compact then of course it is natural to take $\phi \equiv 1$ in $\left(2^{\prime}\right)$. Taking $F$ as given by Lemma 2, (7) implies

$$
\sum_{\alpha=1}^{3} A^{\prime \prime}\left(f_{\alpha}\right) \leq 8 \pi(\gamma+1)-\int_{\widetilde{M}}|B|^{2}+\operatorname{Ric}(\nu) .
$$

Now, as in [SY, p139], we have

$$
|B|^{2}+\operatorname{Ric}(\nu)=-2 K+\operatorname{Ric}\left(\tau_{1}\right)+\operatorname{Ric}\left(\tau_{2}\right),
$$


where $K$ is the Gauss curvature of $\widetilde{M}$. Therefore, by the Gauss-Bonnet theorem,

$$
\sum_{\alpha=1}^{3} A^{\prime \prime}\left(f_{\alpha}\right) \leq 16 \pi-\int_{\widetilde{M}} \operatorname{Ric}\left(\tau_{1}\right)+\operatorname{Ric}\left(\tau_{2}\right) .
$$

It is clear that for this inequality to help, we need a strong condition on the curvature of $N$; taking $\kappa>0$ and assuming the Ricci curvature of $N$ is at least $2 \kappa$, we have

$$
|M|>\frac{2 \pi}{\kappa} \quad \Longrightarrow \quad M \text { is unstable, }
$$

where $|M|$ is the area of $M$.

If $N=\mathbb{S}^{3}$ (so $\kappa=1$ ), we see that $M$ is unstable if $|M|>2 \pi$. In fact, for any minimal $M \subset \mathbb{S}^{3},|M| \geq 4 \pi$ with equality iff $M$ is a totally geodesic $\mathbb{S}^{2}$ (see [LY, Cor10] and Lemma 5 below). This gives a special case of [Sm, Th5.1.1]. Extending the argument slightly, we obtain a new result:

Proposition 3. Suppose $M$ is a compact minimal surface in $\mathbb{R P}^{3}$. Then $M$ is stable iff $M$ is a totally geodesic $\mathbb{R P}^{2}$.

Proof. If $M$ is orientable then $M$ is trivially unstable (take $\phi \equiv 1$ in (2)). Suppose $M$ is nonorientable. Since $\pi_{1}\left(\mathbb{R P}^{3}\right)=\mathbb{Z}_{2}$, the induced map $\pi_{1}(M) \rightarrow \pi_{1}\left(\mathbb{R P}^{3}\right)$ on the fundamental groups either is trivial or gives rise to an index 2 subgroup of $\pi_{1}(M)$. By standard covering theory $([\mathrm{Mu}, 8.14 .2,8.14 .4])$, there is a surface $\widehat{M} \subset \mathbb{S}^{3}$ which either singly or doubly covers $M$. In the former case $|M|=|\widehat{M}| \geq 4 \pi$, and so $M$ is unstable. In the latter case we obtain the same contradiction unless $\widehat{M}$ is a totally geodesic $\mathbb{S}^{2}$, implying $M$ is a totally geodesic $\mathbb{R P}^{2}$. Finally, it is easy to see that such an $\mathbb{R P}^{2}$ is in fact stable: one considers (2) on the double cover and applies, for example, [BCE, 3.4, 3.5].

\section{Remarks.}

(a) [La, Cor9.1] shows that $\mathbb{R}^{3}$ contains many minimal surfaces, both orientable and nonorientable.

(b) The above arguments are related to the (more involved) analysis of stable constant mean curvature surfaces in spherical space forms, given in [RR]. [FR] continues the work of $[\mathrm{RR}]$ and $[\mathrm{EI}]$, using the notion of conformal volume. In particular, $[\mathrm{FR}]$ uses not only conformal maps into $\mathbb{S}^{l}$ but also isometric immersions of the ambient space $N$ into $\mathbb{S}^{l}$ (see [FR, pp372-374]). There seems to be no analogous application of such maps in the nonorientable setting.

(c) The problem of classifying the stable surfaces in other spherical space forms seems to be difficult. By [A, Lemma1], stable (even just minimal) projective planes cannot arise. On the other hand, quotients of the Clifford torus will give rise to flat embedded Klein bottles in the lens spaces $\mathbb{L}(4 n, 1)$ for $n \geq 1$ (see [La, $\S 9]$ ), and these quotients will be stable by [RR, Cor5]. More generally, the following argument (due to H. Rubinstein) shows that for any $g \geq 1$ there is a lens space $\mathbb{L}$ containing a stable embedded nonorientable surface of genus $g$. (Writing a nonorientable surface $S$ as a connected sum of projective planes, the genus of $S$ is defined to be the number of projective planes needed.) Fix $\mathbb{L}=\mathbb{L}(2 k, q)$ with $\operatorname{gcd}(2 k, q)=1$ and let $S \subset \mathbb{L}$ be an embedded nonorientable surface of smallest possible genus $g \equiv N(2 k, q)$. $S$ is incompressible ([H, Lemma 6.3]) and so, by [MSY, Th.1], $S$ is isotopic to an embedded stable 
nonorientable surface (necessarily of the same genus $N(2 k, q)$ ). Allowing $k$ and $q$ to vary, [BW, Th.8.3] shows that $N(2 k, q)$ can take on any positive integer.

Another interesting special case is that of the quotients of triply-periodic minimal surfaces in $\mathbb{R}^{3}$ (so $N=\mathbb{R}^{3} / L$ where $L$ is a discrete lattice of rank 3 ). Here, nontrivial stable surfaces exist. For example, nonorientable quotients of the genus 3 Schwarz $P$ and $D$ surfaces are stable, as are certain perturbations of these surfaces ([R2, Th1, p193]). We do not know if stable higher genus examples exist: $\left(2^{\prime}\right)$ cannot be used here to prove results of great generality, though it can be used to deduce the instability of many known surfaces (i.e. those permitting better estimates than (6)).

It is also worth noting that sufficiently stretched versions of the $D$ surface are unstable, this following from a direct test-function argument using the ideas of [R2, $\S 4]$. However, the instability of these surfaces cannot be deduced by an application of $\left(2^{\prime}\right)$ with Lemma 2 . This provides strong evidence that satisfying $\left(2^{\prime}\right)$ for all $F$, though necessary for stability, is not sufficient.

\section{Nonorientable Cones in $\mathbb{R}^{4}$}

If $M$ is a compact surface in $\mathbb{S}^{3} \subset \mathbb{R}^{4}$, then we define the cone $C(M)$ over $M$ by

$$
C(M)=\mathbb{R}^{4} \cap\{r p: r \geq 0, p \in M\} .
$$

$C(M)$ will be singular at the origin (unless $M=\mathbb{S}^{2}$, implying $C(M)=\mathbb{R}^{3}$ ) but will elsewhere be a regular immersion or embedding as $M$ is. Also, it is easy to see that $C(M)$ is minimal iff $M$ is ([Sm, Prop6.1.1]).

Suppose now that $M$ is minimal and orientable. If $M=\mathbb{S}^{2}$ then $C(M)$ is trivially stable. [A, Lemmas1,2] proved that in any other case $C(M)$ is unstable. It is our intention here to extend that result to nonorientable $M$ by applying $\left(2^{\prime}\right)$ to the orientable double cover $C(\widetilde{M})$. To do this, note that Lemma 2 provides us with an antisymmetric function $F$ on $\widetilde{M}$ and that we can extend $F$ naturally to $C(\widetilde{M})$ by setting $F^{c}(p, r) \equiv F(p)$. Now, mimicking [A], we consider a radial function $\phi=\phi(r)$ on $C(M)$ with $\operatorname{spt}(\phi) \subset \subset C(M)-\{0\}$. With these choices, $\left(2^{\prime}\right)$ gives

$$
\sum_{\alpha=1}^{3} A^{\prime \prime}\left(f_{\alpha} \widetilde{\phi}\right)=2 \int_{C(M)}\left(\phi^{\prime}\right)^{2}+\left(\left|\nabla^{c} F\right|^{2}-\left|B^{c}\right|^{2}\right) \phi^{2},
$$

where $B^{c}$ and $\nabla^{c}$ are, respectively, the second fundamental form and Riemannian connection on $C(M)$. Clearly

$$
\left|\nabla^{c} F^{c}\right|^{2}(p, r)=\frac{1}{r^{2}}|\widetilde{\nabla} F|^{2}(p)
$$

and, by [Sm, Lemma6.1.1] and (8),

$$
\left|B^{c}\right|^{2}(p, r)=\frac{1}{r^{2}}\left|B^{M}\right|^{2}(p)=\frac{2}{r^{2}}(1-K) .
$$


In the same manner the metric on $C(M)$ can be compared to that on $M$, and we find that

$$
\begin{aligned}
\sum_{\alpha=1}^{3} A^{\prime \prime}\left(f_{\alpha} \widetilde{\phi}\right) & =2 \int_{C(M)}\left(\phi^{\prime}\right)^{2}+\frac{1}{r^{2}}\left(|\widetilde{\nabla} F|^{2}+2 K-2\right) \phi^{2} \\
& =2|M| \int_{0}^{\infty} r^{2}\left(\phi^{\prime}\right)^{2}+2\left(\int_{\widetilde{M}}|\widetilde{\nabla} F|^{2}+2 K-2\right) \int_{0}^{\infty} \phi^{2} \\
& \leq 2|M| \int_{0}^{\infty}\left(r^{2}\left(\phi^{\prime}\right)^{2}-2 \phi^{2}\right)+16 \pi \int_{0}^{\infty} \phi^{2},
\end{aligned}
$$

where we have used the Gauss-Bonnet theorem and (7) in the last step.

To continue the argument, we need to show that for suitable $\phi$ the first integral in (9) is negative and we need a suitable lower bound on $|M|$. These facts are provided by the following two lemmas:

Lemma 4 ([Sm, Lemma 6.1.5]). For any $c>\frac{1}{4}$ there exists a smooth $\phi:[0, \infty) \rightarrow$ $\mathbb{R}$ with $\operatorname{spt}(\phi) \subset \subset(0, \infty)$ and for which

$$
\int_{0}^{\infty} r^{2}\left(\phi^{\prime}\right)^{2}<c \int_{0}^{\infty} \phi^{2} .
$$

Lemma 5 ([LY, Corollary 10], and see below). Suppose $M$ is a compact minimal surface in $\mathbb{S}^{3}$. Then $|M| \geq 4 \pi$ with equality iff $M$ is a totally geodesic $\mathbb{S}^{2}$. If $M$ is nonorientable then $|M|>8 \pi$.

Combining (9), Lemma 4 (with $c=1$ ) and Lemma 5, we immediately have the desired result:

Proposition 6. Suppose $M$ is a compact nonorientable minimal surface in $\mathbb{S}^{3}$. Then the cone $C(M)$ over $M$ is unstable.

Proof of Lemma 5. The first part of Lemma 5 is well-known, but this is perhaps not so true for the second part on nonorientable $M$ (notice that we do in fact need the stronger estimate to establish Proposition 6): [LY, Cor10] gives a proof of both results, the argument involving the notion of conformal volume (a related argument is given in [K1, Prop1.3, Fact1.5]). There is also a short proof of Lemma 5 using geometric measure theory, well known in the field, and we would like to end this section by presenting that argument here. (The following can be done in great generality, but we will stay within the context of our desired application).

Suppose $M^{\prime}$ is a 3 -dimensional minimal "surface" in $\mathbb{R}^{4}$. For $p \in \mathbb{R}^{4}$ and $r>0$ consider the volume ratio

$$
D_{r}\left(M^{\prime}, p\right) \equiv \frac{\left|M^{\prime} \cap B_{r}(p)\right|}{\frac{4}{3} \pi r^{3}},
$$

where $B_{r}(p)$ is the open ball in $\mathbb{R}^{4}$ of radius $r$ about $p$. Suppose $R>0, M^{\prime}$ has no boundary in $B_{R}(p)$ and $\left|M^{\prime} \cap B_{r}(p)\right|<\infty$. Then the monotonicity formula ([Si2, 17.3]) states that $D_{r}\left(M^{\prime}, p\right)$ is a monotonically increasing function of $r$ for 
$0<r<R$; this result holds even if $M^{\prime}$ is quite singular, in particular if $M^{\prime}=C(M)$ is a cone over a smooth, compact, minimally immersed surface in $\mathbb{S}^{3}$ (for any $r$ and $p$, because the underlying integral characterization of minimality, [Si2, 16.3], is everywhere valid).

Given $M^{\prime}=C(M)$, we have the inequality

$$
D_{r}(C(M), p) \leq \frac{\left|C(M) \cap B_{r+|p|}(0)\right|}{\frac{4}{3} \pi r^{3}}=\frac{(r+|p|)^{3}}{4 \pi r^{3}}|M|, \quad p \in \mathbb{R}^{4}, r>0 .
$$

Now let $p \in M$ be a point around which $M$ is an embedding. Then $D_{r}(C(M), p)$ $\approx 1$ for small $r$ and thus, by monotonicity, $D_{r}(C(M), p) \geq 1$ for all $r$. Letting $r \rightarrow \infty$ in (11), we find that $|M| \geq 4 \pi$. Equality can only occur if $D_{r}(C(M), p) \equiv 1$, implying $C(M)$ is a cone about $p([\mathrm{Si2}, 19.3])$ : it then follows that $C(M)$ is a plane and $M$ must be a totally geodesic $\mathbb{S}^{2}$.

Finally, if $M \subset \mathbb{S}^{3}$ is nonorientable then $M$ must have non-trivial self-intersection. Thus, taking suitable $p \in M$, we have $D_{r}(C(M), p) \approx k \geq 2$ for small $r$. Repeating the above argument, we find that $|M| \geq 8 \pi$, and here it is clear that equality is impossible.

\section{Noncompact Surfaces in 3-Manifolds of Nonnegative Scalar Curvature}

Suppose $N$ is a 3-manifold with nonnegative scalar curvature $S$, and suppose $M$ is a complete and noncompact nonorientable minimal surface in $P$. By (8),

$$
|B|^{2}+\operatorname{Ric}(\nu)=\frac{1}{2}|B|^{2}+S-K \geq \frac{1}{2}|B|^{2}-K \quad \text { on } M .
$$

Therefore, if $M$ is stable and if $F: \widetilde{M} \rightarrow \mathbb{S}^{2}$ is an antisymmetric function on the orientable double cover, then, by $\left(2^{\prime}\right)$,

$$
\int_{M}\left|\nabla^{M} \phi\right|^{2}+\left(K-\frac{1}{2}|B|^{2}+|\widetilde{\nabla} F|^{2}\right) \phi^{2} \geq 0
$$

for any smooth compactly supported $\phi: M \rightarrow \mathbb{R}$. [GL, §3] analyses a surface $M$ under the assumption that, for some constant $\beta>\frac{1}{2}$, the operator $\Delta^{M}-\beta K$ is positive. Our intention is to use in (12) the inequalities obtained there.

Following [GL], let $\Omega_{0} \subset M$ be a (rather arbitrary) compact set with analytic boundary, and define $s(x)=\operatorname{dist}\left(x, \Omega_{0}\right)$. For $R>0$ let $\phi_{R}(x)=\psi\left(\frac{s(x)}{R}\right)$, where $\psi(t)$ is a smooth approximation to $\max (1-t, 0)$. By [GL, 3.13, $\beta=1]$ and (12)

$$
\frac{1}{2} \int_{M}|B|^{2} \phi_{R}^{2}+\frac{1}{R^{2}}\left|\Omega_{R}-\Omega_{0}\right| \leq 2 \pi+\frac{2}{R^{2}} L(0)+\int_{M}|\widetilde{\nabla} F|^{2} \phi_{R}^{2},
$$

where $\Omega_{R}=M \cap\{x: s(x)=R\}$ and $L(0)$ is the length of $\partial \Omega_{0}$. If $F$ can be chosen to have finite energy, then we can let $R \rightarrow \infty$ :

Proposition 7. Suppose $M$ is a complete and noncompact, nonorientable stable minimal surface in a 3-manifold $N$ of nonnegative scalar curvature. If the orientable double cover $\widetilde{M}$ of $M$ admits an antisymmetric map $F: \widetilde{M} \rightarrow \mathbb{S}^{2}$ of finite energy then $M$ has quadratic area growth and $\int_{M}|B|^{2}<\infty$. 
Remark. Note that when $N=\mathbb{R}^{3}$ this result is complemented by [R1, Th3]. We discuss this further below.

Suppose $\Omega \subset \Omega^{\prime}$ are piecewise smooth compact nonorientable subdomains of $M$. Then $\Omega^{\prime}$ has no smaller genus than $\Omega$. We can therefore define the (possibly infinite) genus of $M$.

Suppose in fact that genus $M=\gamma<\infty$. Let $R>0$ be such that $\partial \Omega_{R}$ is piecewise smooth (which will be true for most $R$ - see [GL, p224]). By smoothly capping the ends of $\Omega_{R}$ and taking the orientable double cover, we obtain a compact Riemann surface $\bar{\Omega}_{R}$ of genus at most $\gamma-1$. Lemma 2 provides an antisymmetric $F_{R}: \bar{\Omega}_{R} \rightarrow \mathbb{S}^{2}$ with

$$
\int_{\Omega_{R}}\left|\widetilde{\nabla} F_{R}\right|^{2} \leq \frac{1}{2} \int_{\bar{\Omega}_{R}}\left|\widetilde{\nabla} F_{R}\right|^{2} \leq 4 \pi \gamma .
$$

One can now use weak compactness in the Sobolev spaces $H^{1,2}\left(\Omega_{R}, \mathbb{S}^{2}\right)$, diagonalizing on $R$ to obtain an $F$ on the entire double cover of $M$ which satisfies the same estimate (14). Alternatively, since $\left|\phi_{R}\right| \leq 1$, we can use (14) directly in (13) and then let $R \rightarrow \infty$. Using either method, we find

Proposition 8 ([LS]). The conclusions of Proposition 7 continue to hold if the hypothesis of the existence of a finite energy map is replaced by the hypothesis that $M$ has finite genus.

Final Remark. Of course, without some special hypothesis there is no reason to expect that $M$ will admit an antisymmetric $F$ of finite energy: the problem in general, then, is to show that $F$ can be chosen to have suitably slow energy growth. Though plausible, this appears to be difficult.

\section{ACKNOWLEDGMENTS}

I would like to thank Hyam Rubinstein and Brian White for some very informative discussions. Thanks also to Lene and Rie for their inestimable contribution.

\section{REFERENCES}

[A] F.J. Almgren, Jr., Some interior regularity theorems for minimal surfaces and an extension of Bernstein's theorem, Ann. of Math. 85 (1966), 277-292. MR 34:702

[BCE] J.L. Barbosa, M.P. do Carmo and J. Eschenburg, Stability of hypersurfaces of constant mean curvature in Riemannian manifolds, Math. Zeit. 197 (1988), 123-138. MR 88m:53109

[BW] G. E. Bredon and J. W. Wood, Non-orientable surfaces in orientable 3-manifolds, Invent. Math. 7 (1969), 83-110. MR 39:7616

[Ca1] M.P. doCarmo, Stability of minimal submanifolds, Global Differential Geometry and Global Analysis, Lect. Notes in Math. 838, Springer-Verlag, Berlin, 1981, pp. 129-139. MR 82k:53073

[Ca2] _ Riemannian Geometry, Birkhäuser, Boston, 1992. MR 92i:53001

[Ch] J. Choe, Index, vision number and stability of complete minimal surfaces, Arch. Rational Mech. Anal. 109 (1990), 195-212. MR 91b:53007

[EI] A. El Soufi and S. Ilias, Majoration de la seconde valeur propre d'un operateur de Schrödinger sur une variete compacte et applications, J. Funct. Anal. 103 (1992), 294-316. MR 93g:58150 
[F] D. Fischer-Colbrie, On complete minimal surfaces with finite Morse index in three manifolds, Invent. Math. 82 (1985), 121-132. MR 87b:53090

[FR] S. Fornari and J. Ripoll, Stability of compact hypersurfaces with constant mean curvature, Indiana Univ. Math. Jour. 43 (1994), 367-381. MR 95g:53075

[FS] D. Fischer-Colbrie and R. Schoen, The structure of complete stable minimal surfaces in 3manifolds of non-negative scalar curvature, Comm. Pure Appl. Math. 33 (1980), 199-211. MR 81i:53044

[GH] P. Griffiths and J. Harris, Principles of Algebraic Geometry, Wiley, New York, 1978. MR 80b: 14001

[GL] R. Gulliver and H. B. Lawson, The structure of stable minimal hypersurfaces near a singularity, Proc. Symp. Pure Math., A.M.S. 44 (1986), 213-239. MR 87g:53091

[H] J. Hempel, 3-Manifolds, Annals of Math. Studies 86, Princeton Univ. Press, New Jersey, 1976. MR 54:3702

[K1] R. Kusner, Conformal geometry and complete minimal surfaces, Bull. Amer. Math. Soc. 17 (1987), 296-300. MR 88j:53008

[K2] _ Comparison surfaces for the Willmore problem, Pac. J. Math. 138 (1989), 317345. MR 90e:53013

[La] H.B. Lawson, Complete minimal surfaces in $\mathbb{S}^{3}$, Ann. of Math. 92 (1970), 335-374. MR 42:5170

[LaS] H.B. Lawson and J. Simons, On stable currents and their applications to global problems in real and complex geometry, Ann. of Math. 98 (1973), 427-450. MR 48:2881

[LiS] I.C. Lima and A.M. da Silveira, Stability of complete nonorientable minimal surfaces in $\mathbf{R}^{3}$, preprint.

[LR] F.J. Lopez and A. Ros, Complete minimal surfaces with index one and stable constant mean curvature surfaces, Comm. Math. Helv. 64 (1989), 34-43. MR 90b:53006

[LY] P. Li and S.-T. Yau, A new conformal invariant and its applications to the Willmore conjecture and the first eigenvalue of compact surfaces, Invet. Math. 69 (1982), 269-291. MR 84f: 53049

[Ma] G. Martens, Minimale Blätterzahl bei Überlagerungen Kleinscher Flächen über der projectiven Ebene, Arch. Math. 30 (1978), 481-486. MR 58:16697

[MSY] W. Meeks III, L. Simon and S.-T. Yau, Embedded minimal surfaces, exotic spheres and manifolds with positive Ricci curvature, Ann. of Math. 116 (1982), 621-659. MR 84f:53053

[Mi] M.J. Micallef, Stable minimal surfaces in Euclidean space, J. Diff. Geom. 19 (1984), 64-76. MR 85e:53009

[Mu J.R. Munkres, Topology, Prentice-Hall, Eaglewood Cliffs, New Jersey, 1975. MR 57:4063

[P] B. Palmer, Stability of minimal hypersurfaces, Comm. Math. Helv. 66 (1991), 185-188. MR 92m:58023

[R1] M. Ross, Complete nonorientable minimal surfaces in $\mathbf{R}^{3}$, Comm. Math. Helv. 67 (1992), 64-76. MR 92k:53022

[R2] M. Ross, Schwarz' P and D surfaces are stable, Diff. Geom. Appl. 2 (1992), 179-195. MR 94j:53010

[RR] M. Ritore and A. Ros, Stable constant mean curvature tori and the isoperimetric problem in three space forms, Comm. Math. Helv. 67 (1992), 293-305. MR 93a:53055

[RS] M. Ross and C. Schoen, Stable quotients of periodic minimal surfaces, Comm. Anal. Geom. 2 (1994), 451-459. MR 95j:53016

[Sh] K. Shiohama, Total curvatures and minimal areas of complete surfaces, Proc. A.M.S. 94 (1985), 310-316. MR 86h:53047

[Si1] L. Simon, First and second variation in geometry and topology, University of Melbourne Research Report, 1979.

[Si2] Lectures on Geometric Measure Theory, Proc. Centre Math. Anal., Aust. Nat. Univ., Canberra, Australia, 1984. MR 87a:49001

[Sm] J. Simons, Minimal varieties in Riemannian manifolds, Ann. of Math. 88 (1968), 62-105. MR 38:1617

[Sy] J.L. Synge, On the connectivity of spaces of positive curvature, Quart. J. Math. (Oxford Series) 7 (1936), 316-320.

[SY] R. Schoen and S.-T. Yau, Existence of incompressible minimal surfaces and the topolgy of three-dimensional manifolds with non-negative scalar curvature, Ann. of Math. 110 (1979), 127-142. MR 81k:53029 
[Ya] S.-T. Yau, Nonlinear analysis in geometry, L'Enseignement Math. 33 (1987), 109-158. MR 88g:58003; MR 88e:53001

[Yp] S. Yaprak, Bernstein type theorems for minimal surfaces, Geometry and Toplogy of Submanifolds IV, World Scientific, River Edge, New Jersey, 1992, pp. 30-42. MR 93j:53011

Department of Mathematics, Melbourne University, Parkville, Victoria, 3052, AusTRALIA

E-mail address: marty@mundoe.maths.mu.oz.au

Current address: Antarctic CRC, Box 252-80, Hobart, Tasmania, Australia 